\title{
Ligand, Copper, and Amine-Free Sonogashira Reaction of Aryl Iodides and Bromides with Terminal Alkynes
}

Sameer Urgaonkar and John G. Verkade*

Department of Chemistry, Gilman Hall, Iowa State University, Ames, IA, 50011

jverkade@iastate.edu

\section{SUPPORTING INFORMATION}

\author{
TABLE OF CONTENTS
}

Contents

1) General considerations and experimental procedure

2) Spectroscopy Data

3) References for known compounds
Page number

S2-S3

S3-S8

S8 


\section{General Considerations:}

$\mathrm{Pd}(\mathrm{OAc})_{2}$ and tetrabutylammonium acetate were purchased from Aldrich and used without further purification. Anhydrous DMF was purchased from Aldrich in Sure/Seal ${ }^{\circledR}$ bottles and dispensed by syringe. All other reagents were commercially available and used without further purification. All reactions were performed under an atmosphere of argon in oven-dried glassware. ${ }^{1} \mathrm{H}$ and ${ }^{13} \mathrm{C}$ NMR spectra were recorded at 300 and 75 $\mathrm{MHz}$, respectively, unless otherwise noted. Alumina gel flash chromatography was performed using Fluka Basic Brockman Activity I Alumina 60-325 mesh. The yields reported are isolated yields and are the average of two runs. Compounds known in the literature were characterized by comparing their ${ }^{1} \mathrm{H}$ and ${ }^{13} \mathrm{C}$ NMR or mass spectra to the previously reported data. In all cases, the comparisons were very favorable. New compounds were characterized by elemental analysis, ${ }^{1} \mathrm{H}$ and ${ }^{13} \mathrm{C}$ NMR spectroscopy, and high resolution mass spectrometry.

\section{General Procedure for Ligand, Copper, and Amine-Free Sonogashira Coupling of Aryl Iodides and Bromides with Terminal Alkynes.}

An oven-dried Schlenk flask equipped with a magnetic stirring bar was charged with $\mathrm{Bu}_{4} \mathrm{NOAc}(1.5 \mathrm{mmol})$ and $\mathrm{Pd}(\mathrm{OAc})_{2}(1-3 \mathrm{~mol} \%)$ or $\mathrm{Pd}_{2}(\mathrm{dba})_{3}(2 \mathrm{~mol} \%$ for aryl bromides) inside a nitrogen-filled glove box. The flask was capped with a rubber septum and then it was removed from the glove box. An aryl iodide or bromide $(1.0 \mathrm{mmol})$ and then DMF (3 mL) were then added and after 5 min of stirring, the alkyne $(1.0 \mathrm{mmol})$ was

added. Stirring was continued at room temperature under argon for the corresponding reaction times indicated in the Tables, after which the reaction mixture was diluted with water $(10 \mathrm{~mL})$ and extracted with diethyl ether $(4 \mathrm{x} 10 \mathrm{~mL})$. The combined ether layers 
were dried over $\mathrm{Na}_{2} \mathrm{SO}_{4}$, filtered, concentrated, and purified by alumina gel flash chromatography using hexanes or hexanes/ether to elute the desired coupling product.

\section{Spectroscopy Data:}

4-Ethoxycarbonyldiphenylacetylene ${ }^{1}$ (Table 1, entry 1) ${ }^{1} \mathrm{H}$ NMR $\left(\mathrm{CDCl}_{3}\right)$ : $\delta$ 8.05-8.02 (m, 2H), 7.60-7.53 (m, 4H), 7.38-7.26 (m, 3H), $4.42(\mathrm{q}, J=7.2 \mathrm{~Hz}, 2 \mathrm{H}), 1.41$ (t, $J=7.2$ $\mathrm{Hz}, 3 \mathrm{H}) .{ }^{13} \mathrm{C}$ NMR $\left(\mathrm{CDCl}_{3}\right): \delta 166.3,131.9,131.7,130.0,129.7,129.0,128.7,128.1$, $122.9,92.5,88.9,61.4,14.6$.

4-(Phenylethynyl)acetophenone ${ }^{2}$ (Table 1, entry 2) ${ }^{1} \mathrm{H}$ NMR $\left(\mathrm{CDCl}_{3}\right): \delta$ 7.94-7.91 (m, 2H), 7.61-7.53 (m, 4H), 7.37-7.35 (m, 3H), $2.59(\mathrm{~s}, 3 \mathrm{H}) .{ }^{13} \mathrm{C}$ NMR $\left(\mathrm{CDCl}_{3}\right): \delta$ 197.5, 136.4, 132.0, 131.9, 129.1, 128.7, 128.5, 128.4, 122.9, 93.0, 88.9, 26.8.

4-Nitrodiphenylacetylene ${ }^{2}$ (Table 1, entry 3) ${ }^{1} \mathrm{H}$ NMR $\left(\mathrm{CDCl}_{3}\right): \delta 8.23-8.20(\mathrm{~m}, 2 \mathrm{H})$, 7.68-7.65 (m, 2H), 7.58-7.55 (m, 2H), 7.40-7.39 (m, 3H). ${ }^{13} \mathrm{C}$ NMR $\left(\mathrm{CDCl}_{3}\right): \delta$ 147.2, $132.5,132.1,130.5,129.5,128.8,123.9,122.3,94.9,87.8$.

2-Methyldiphenylacetylene ${ }^{2}$ (Table 1, entry 4) ${ }^{1} \mathrm{H} \mathrm{NMR}\left(\mathrm{CDCl}_{3}\right): \delta$ 7.61-7.54 (m, 3H), 7.43-7.37 (m, 3H), 7.29-7.19 (m, 3H), 2.57 (s, 3H). $\left.{ }^{13} \mathrm{C} \mathrm{NMR} \mathrm{(CDCl} 3\right): \delta 140.4,132.1$, $131.8,129.7,128.6,128.5,128.4,125.9,123.8,123.3,93.6,88.6,21.0$.

3-Methoxydiphenylacetylene ${ }^{3}$ (Table 1, entry 5) ${ }^{1} \mathrm{H}$ NMR $\left(\mathrm{CDCl}_{3}\right): \delta$ 7.56-7.51 (m, 2H), 7.41-7.33 (m, 3H), 7.29-7.24 (m, 1H), 7.16-7.13 (m, 1H), 7.08-7.07 (m, 1H), 6.92$6.88(\mathrm{~m}, 1 \mathrm{H}), 3.83(\mathrm{~s}, 3 \mathrm{H}) .{ }^{13} \mathrm{C} \mathrm{NMR}\left(\mathrm{CDCl}_{3}\right): \delta 159.6,131.9,129.6,128.6,128.5,124.5$, $124.4,123.4,116.5,115.2,89.5,89.4,55.5$.

2-Methoxydiphenylacetylene ${ }^{2}$ (Table 1, entry 6) ${ }^{1} \mathrm{H}$ NMR $\left(\mathrm{CDCl}_{3}\right): \delta$ 7.59-7.50 (m, 3H), 7.38-7.29 (m, 4H), 6.98-6.90 (m, 2H), $3.92(\mathrm{~s}, 3 \mathrm{H}) .{ }^{13} \mathrm{C}$ NMR $\left(\mathrm{CDCl}_{3}\right): \delta$ 160.1, $133.8,131.9,130.0,128.5,128.3,123.8,120.7,112.7,110.9,93.7,85.9,56.1$. 
4-Methoxydiphenylacetylene ${ }^{2}$ (Table 1, entry 7) ${ }^{1} \mathrm{H}$ NMR $\left(\mathrm{CDCl}_{3}\right): \delta$ 7.55-7.47 (m, 4H), 7.38-7.32 (m, 3H), 6.91-6.88 (m, 2H), $3.83(\mathrm{~s}, 3 \mathrm{H}) .{ }^{13} \mathrm{C} \mathrm{NMR}\left(\mathrm{CDCl}_{3}\right): \delta$ 159.9, $133.3,131.7,128.5,128.2,123.8,115.6,114.2,89.6,88.3,55.5$.

1-(4-Cyclohex-1-enylethynylphenyl)-ethanone ${ }^{2}$ (Table 2, entry 1) ${ }^{1} \mathrm{H} \mathrm{NMR}\left(\mathrm{CDCl}_{3}\right): \delta$ 7.88-7.85 (m, 2H), 7.48-7.45 (m, 2H), 6.27-6.23 (m, 1H), $2.57(\mathrm{~s}, 3 \mathrm{H}), 2.24-2.12(\mathrm{~m}$, $4 \mathrm{H}), 1.71-1.57(\mathrm{~m}, 4 \mathrm{H}) .{ }^{13} \mathrm{C}$ NMR $\left(\mathrm{CDCl}_{3}\right): \delta$ 197.5, 136.8, 135.9, 131.7, 129.0, 128.4, $120.7,95.0,86.4,29.3,26.8,26.1,22.5,21.6$.

1-(4'-Acetylphenyl)-1-octyne ${ }^{2}$ (Table 2, entry 2$)^{1}{ }^{1} \mathrm{H}$ NMR $\left(\mathrm{CDCl}_{3}\right): \delta$ 7.87-7.85 (m, 2H), 7.46-7.43 (m, 2H), 2.57 (s, 3H), 2.44-2.39 (m, 2H), 1.65-1.56 (m, 2H), 1.49-1.40 (m, 2H), 1.33-1.29 (m, 4H), 0.92-0.87 (m, 3H). ${ }^{13} \mathrm{C} \mathrm{NMR}\left(\mathrm{CDCl}_{3}\right): \delta$ 197.6, 135.8, 131.8, $129.4,128.4,94.7,80.3,31.6,28.8,28.7,26.8,22.8,19.8,14.3$.

4-Nitro-1-oct-1-ynyl-benzene ${ }^{2}$ (Table 2, entry 3) ${ }^{1} \mathrm{H}$ NMR $\left(\mathrm{CDCl}_{3}\right): \delta$ 8.14-8.11 (m, $2 \mathrm{H}), 7.50-7.47(\mathrm{~m}, 2 \mathrm{H}), 2.44-2.40(\mathrm{~m}, 2 \mathrm{H}), 1.65-1.55(\mathrm{~m}, 2 \mathrm{H}), 1.48-1.28(\mathrm{~m}, 6 \mathrm{H}), 0.89$ (m, 3H). ${ }^{13} \mathrm{C}$ NMR $\left(\mathrm{CDCl}_{3}\right): \delta$ 146.7, 132.4, 131.4, 123.7, 97.0, 79.5, 31.5, 28.8, 28.6, $22.8,19.8,14.3$.

4-(5-Hydroxy-hex-1-ynyl)-nitrobenzene ${ }^{4}$ (Table 2, entry 4) ${ }^{1} \mathrm{H}$ NMR $\left(\mathrm{CDCl}_{3}\right): \delta 8.12-$ $8.09(\mathrm{~m}, 2 \mathrm{H}), 7.49-7.46(\mathrm{~m}, 2 \mathrm{H}), 3.68(\mathrm{t}, J=6.0 \mathrm{~Hz}, 2 \mathrm{H}), 2.49-2.45(\mathrm{~m}, 2 \mathrm{H}), 1.94(\mathrm{bs}$, 1H), 1.76-1.66 (m, 4H). ${ }^{13} \mathrm{C}$ NMR $\left(\mathrm{CDCl}_{3}\right): \delta 146.8,132.4,131.2,123.7,96.4,62.4$, $32.0,25.0,19.6$.

1-Cyclohex-1-enylethynyl-4-nitrobenzene ${ }^{2}$ (Table 2, entry 5) ${ }^{1} \mathrm{H}$ NMR $\left(\mathrm{CDCl}_{3}\right): \delta$ $8.15(\mathrm{~d}, J=8.8 \mathrm{~Hz}, 2 \mathrm{H}), 7.52(\mathrm{~d}, J=8.8 \mathrm{~Hz}, 2 \mathrm{H}), 6.32-6.29(\mathrm{~m}, 1 \mathrm{H}), 2.23-2.16(\mathrm{~m}, 4 \mathrm{H})$, 1.73-1.61 (m, 4H). ${ }^{13} \mathrm{C} \mathrm{NMR}\left(\mathrm{CDCl}_{3}\right): \delta 146.8,137.9,132.2,131.1,123.8,120.4,97.1$, $85.5,29.1,26.1,22.4,21.6$. 
2-Methyl-1-oct-1-ynyl-benzene ${ }^{2}$ (Table 2, entry 6) ${ }^{1} \mathrm{H}$ NMR $\left(\mathrm{CDCl}_{3}\right): \delta 7.43-7.40(\mathrm{~m}$, $1 \mathrm{H}), 7.22-7.11(\mathrm{~m}, 3 \mathrm{H}), 2.52-2.47(\mathrm{~m}, 5 \mathrm{H}), 1.72-1.62(\mathrm{~m}, 2 \mathrm{H}), 1.58-1.48(\mathrm{~m}, 2 \mathrm{H}), 1.40-$ $1.35(\mathrm{~m}, 4 \mathrm{H}), 0.96(\mathrm{t}, J=6.8 \mathrm{~Hz}, 3 \mathrm{H}) .{ }^{13} \mathrm{C} \mathrm{NMR}\left(\mathrm{CDCl}_{3}\right): \delta 140.1,132.0,129.5,127.7$, $125.6,124.1,94.7,79.7,31.7,29.2,28.9,22.9,21.0,19.8,14.3$.

1-Cyclohex-1-enylethynyl-2-methylbenzene ${ }^{2}$ (Table 2, entry 7) ${ }^{1} \mathrm{H} \mathrm{NMR}\left(\mathrm{CDCl}_{3}\right): \delta$ 7.43-7.40 (m, 1H), 7.21-7.11 (m, 3H), 6.25-6.22 (m, 1H), 2,46 (s, 3H), 2.30-2.15 (m, $4 \mathrm{H}), 1.76-1.61(\mathrm{~m}, 4 \mathrm{H}) .{ }^{13} \mathrm{C} \mathrm{NMR}\left(\mathrm{CDCl}_{3}\right): \delta 140.1,134.9,131.9,129.5,128.0,125.7$, $123.7,121.2,95.6,86.0,29.6,26.0,22.6,21.8,21.0$.

2-(5-Hydroxy-hex-1-ynyl)-methylbenzene (Table 2, entry 8) ${ }^{1} \mathrm{H} \mathrm{NMR}\left(\mathrm{CDCl}_{3}\right): \delta$ 7.38-7.35 (m, 1H), 7.18-7.08 (m, 3H), 3.72-3.68 (m, 2H), 2.52-2.48 (m, 2H), $2.42(\mathrm{~s}$, $3 \mathrm{H}), 1.77-1.65(\mathrm{~m}, 5 \mathrm{H}) .{ }^{13} \mathrm{C} \mathrm{NMR}\left(\mathrm{CDCl}_{3}\right): \delta 140.1,132.0,129.5,127.8,125.7,123.9$, 94.1, 80.0, 62.6, 32.1, 25.4, 21.0, 19.6. HRMS m/z Calcd for $\mathrm{C}_{13} \mathrm{H}_{16} \mathrm{O}: 188.12012$. Found: 188.12030. Anal. Calcd for $\mathrm{C}_{13} \mathrm{H}_{16} \mathrm{O}$ : C, 82.98; H, 8.51. Found: C, 82.70; H, 8.47. 1-Cyclohex-1-enylethynyl-3-methoxybenzene (Table 2, entry 9) ${ }^{1} \mathrm{H}$ NMR $\left(\mathrm{CDCl}_{3}\right): \delta$ 7.23-7.17 (m, 1H), 7.03-7.00 (m, 1H), 6.96-6.95 (m, 1H), 6.85-6.82 (m, 1H), 6.24-6.20 $(\mathrm{m}, 1 \mathrm{H}), 3.79(\mathrm{~s}, 3 \mathrm{H}), 2.17-2.12(\mathrm{~m}, 4 \mathrm{H}), 1.71-1.58(\mathrm{~m}, 4 \mathrm{H}) .{ }^{13} \mathrm{C} \mathrm{NMR}\left(\mathrm{CDCl}_{3}\right): \delta 159.5$, $135.6,129.5,125.0,124.2,120.9,116.3,114.7,91.3,86.9,55.5,29.4,26.0,22.6,21.7$. HRMS $m / z$ Calcd for $\mathrm{C}_{15} \mathrm{H}_{16} \mathrm{O}: 212.12012$. Found 212.1203. Anal. Calcd for $\mathrm{C}_{15} \mathrm{H}_{16} \mathrm{O}: \mathrm{C}$, 84.91; H, 7.55. Found: C, 84.88; H, 7.41.

3-(5-Hydroxy-hex-1-ynyl)-methoxybenzene (Table 2, entry 10) ${ }^{1} \mathrm{H} \mathrm{NMR}\left(\mathrm{CDCl}_{3}\right): \delta$ 7.20-7.14 (m, 1H), 6.99-6.96 (m, 1H), 6.92-6.91 (m, 1H), 6.83-6.79 (m, 1H), $3.76(\mathrm{~s}$, $3 \mathrm{H}), 3.68-3.64(\mathrm{~m}, 2 \mathrm{H}), 2.45-2.40(\mathrm{~m}, 2 \mathrm{H}), 2.18(\mathrm{bs}, 1 \mathrm{H}), 1.77-1.61(\mathrm{~m}, 4 \mathrm{H}) .{ }^{13} \mathrm{C} \mathrm{NMR}$ $\left(\mathrm{CDCl}_{3}\right): \delta 159.5,129.5,125.1,124.3,116.6,114.4,90.1,81.1,62.5,55.4,32.1,25.2$ 
19.4. HRMS $\mathrm{m} / z$ Calcd for $\mathrm{C}_{13} \mathrm{H}_{16} \mathrm{O}_{2}$ : 204.11503. Found: 204.11530. Anal. Calcd for $\mathrm{C}_{13} \mathrm{H}_{16} \mathrm{O}_{2}:$ C, 76.47; H, 7.84. Found: C, 76.31; H, 7.67.

1-Cyclohex-1-enylethynyl-2-methoxybenzene ${ }^{2}$ (Table 2, entry 11) ${ }^{1} \mathrm{H}$ NMR $\left(\mathrm{CDCl}_{3}\right)$ : $\delta$ 7.23-7.18 (m, 1H), 7.04-7.02 (m, 1H), 6.97-6.96 (m, 1H), 6.86-6.82 (m, 1H), 6.24-6.21 (m, $1 \mathrm{H}), 3.79(\mathrm{~s}, 3 \mathrm{H}), 2.24-2.12(\mathrm{~m}, 4 \mathrm{H}), 1.73-1.58(\mathrm{~m}, 4 \mathrm{H}) .{ }^{13} \mathrm{C} \mathrm{NMR}\left(\mathrm{CDCl}_{3}\right): \delta 159.5$, 135.5, 129.5, 125.0, 124.2, 120.9, 116.4, 114.7, 91.3, 86.9, 55.4, 29.5, 26.0, 22.6, 21.8.

2-(5-Hydroxy-hex-1-ynyl)-methoxybenzene (Table 2, entry 12) ${ }^{1} \mathrm{H} \mathrm{NMR}\left(\mathrm{CDCl}_{3}\right): \delta$ 7.37-7.34 (m, 1H), 7.25-7.20 (m, 1H), 6.89-6.82 (m, 2H), $3.85(\mathrm{~s}, 3 \mathrm{H}), 3.71-3.67(\mathrm{~m}$, $2 \mathrm{H}), 2.52-2.48(\mathrm{~m}, 2 \mathrm{H}), 2.41$ (bs, $1 \mathrm{H}), 1.80-1.64(\mathrm{~m}, 4 \mathrm{H}) .{ }^{13} \mathrm{C} \mathrm{NMR}\left(\mathrm{CDCl}_{3}\right): \delta 160.0$ 133.8, 120.6, 113.1, 110.7, 94.5, 82.3, 62.5, 56.0, 32.1, 25.2, 19.7. HRMS $m / z$ Calcd for $\mathrm{C}_{13} \mathrm{H}_{16} \mathrm{O}_{2}$ : 204.11503. Found: 204.11530. Anal. Calcd for $\mathrm{C}_{13} \mathrm{H}_{16} \mathrm{O}_{2}: \mathrm{C}, 76.47 ; \mathrm{H}, 7.84$. Found: C, 76.22; H, 7.77.

2-Methoxy-1-oct-1-ynyl-benzene ${ }^{2}$ (Table 2, entry 13) ${ }^{1} \mathrm{H}$ NMR $\left(\mathrm{CDCl}_{3}\right): \delta$ 7.40-7.37 (m, 1H), 7.26-7.20 (m, 1H), 6.90-6.83 (m, 2H), $3.87(\mathrm{~s}, 3 \mathrm{H}), 2.50-2.45(\mathrm{~m}, 2 \mathrm{H}), 1.68-$ $1.59(\mathrm{~m}, 2 \mathrm{H}), 1.53-1.43(\mathrm{~m}, 2 \mathrm{H}), 1.35-1.31(\mathrm{~m}, 4 \mathrm{H}), 0.94-0.89(\mathrm{~m}, 3 \mathrm{H}) .{ }^{13} \mathrm{C}$ NMR $\left(\mathrm{CDCl}_{3}\right): \delta 160.0,133.8,129.0,120.6,113.4,110.7,94.9,76.9,55.9,31.6,29.1,28.9$, $22.8,20.0,14.3$.

1-(5-Chloro-1-pentynyl)-4-methoxybenzene (Table 2, entry 14) ${ }^{1} \mathrm{H}$ NMR $\left(\mathrm{CDCl}_{3}\right): \delta$ 7.35-7.32 (m, 2H), 6.83-6.80 (m, 2H), $3.79(\mathrm{~s}, 3 \mathrm{H}), 3.73-3.69(\mathrm{~m}, 2 \mathrm{H}), 2.61-2.57(\mathrm{~m}$, 2H), 2.09-2.00 (m, 2H). ${ }^{13} \mathrm{C}$ NMR (100 MHz, $\left.\mathrm{CDCl}_{3}\right): \delta 159.4,133.1,115.9,114.1,86.7$, 81.5, 55.5, 44.1, 31.8, 17.1. HRMS $\mathrm{m} / z$ Calcd for $\mathrm{C}_{12} \mathrm{H}_{13} \mathrm{OCl}$ : 208.06549. Found: 208.06580. Anal. Calcd for $\mathrm{C}_{12} \mathrm{H}_{13} \mathrm{OCl}$ : C, 69.08; H, 6.24. Found: C, 69.38; H, 6.11. 
4-(5-Hydroxy-hex-1-ynyl)-methoxybenzene ${ }^{6}$ (Table 2 , entry 15$){ }^{1} \mathrm{H} \mathrm{NMR}\left(\mathrm{CDCl}_{3}\right)$ : $\delta$ 7.34-7.29 (m, 2H), 6.82-6.77 (m, 2H), $3.78(\mathrm{~s}, 3 \mathrm{H}), 3.71-3.66(\mathrm{~m}, 2 \mathrm{H}), 2.45-2.40(\mathrm{~m}$, $2 \mathrm{H}), 1.80-1.61(\mathrm{~m}, 5 \mathrm{H}) .{ }^{13} \mathrm{C} \mathrm{NMR}\left(\mathrm{CDCl}_{3}\right): \delta 159.2,133.1,116.2,114.0,88.5,80.9$, $62.7,55.5,32.1,25.3,19.4$.

1-Cyclohex-1-enylethynyl-4-methoxybenzene ${ }^{2}$ (Table 2 , entry 16) ${ }^{1} \mathrm{H}$ NMR $\left(\mathrm{CDCl}_{3}\right)$ :

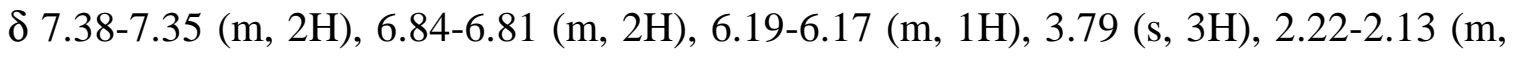
4H), 1.72-1.58 (m, 4H). ${ }^{13} \mathrm{C} \mathrm{NMR}\left(\mathrm{CDCl}_{3}\right): \delta 159.4,134.6,133.0,121.1,116.1,114.1$, $90.1,86.9,55.5,29.6,26.0,22.6 .21 .8$.

2-(4-Methoxyphenyl)-1-ethynyl(trimethyl)silane ${ }^{7}$ (Table $\quad 2$, entry 17$){ }^{1} \mathrm{H}$ NMR $\left(\mathrm{CDCl}_{3}\right): \delta 7.42(\mathrm{~d}, J=8.8 \mathrm{~Hz}, 2 \mathrm{H}), 6.82(\mathrm{~d}, J=8.9 \mathrm{~Hz}, 2 \mathrm{H}), 3.79(\mathrm{~s}, 3 \mathrm{H}), 3.81(\mathrm{~s}, 3 \mathrm{H})$, $1.15(\mathrm{~s}, 21 \mathrm{H}) .{ }^{13} \mathrm{C} \mathrm{NMR}\left(\mathrm{CDCl}_{3}\right): \delta 159.8,133.7,116.0,114.0,107.4,88.8,55.5,18.9$, 11.6.

6-(4-Methoxyphenyl)-hex-5-ynenitrile (Table 2, entry 18) ${ }^{1} \mathrm{H}$ NMR $\left(\mathrm{CDCl}_{3}\right): \delta 7.34$ $(\mathrm{d}, J=8.7 \mathrm{~Hz}, 2 \mathrm{H}), 6.83(\mathrm{~d}, J=8.8 \mathrm{~Hz}, 2 \mathrm{H}), 3.80(\mathrm{~s}, 3 \mathrm{H}), 2.60-2.52(\mathrm{~m}, 4 \mathrm{H}), 1.99-1.89$ $(\mathrm{m}, 2 \mathrm{H}) .{ }^{13} \mathrm{C} \mathrm{NMR}\left(\mathrm{CDCl}_{3}\right): \delta 159.6,133.2,119.5,115.5,114.1,85.6,82.4,55.5,25.0$, 18.8, 16.4. HRMS m/z Calcd for $\mathrm{C}_{13} \mathrm{H}_{13} \mathrm{NO}$ : 199.09971. Found: 199.10020. Anal. Calcd for $\mathrm{C}_{13} \mathrm{H}_{13} \mathrm{NO}$ : C, 78.39; H, 6.53. Found: C, 78.66; H, 6.39.

4-(5-Hydroxy-hex-1-ynyl)-cyanobenzene (Table 3, entry 5) ${ }^{1} \mathrm{H} \mathrm{NMR}\left(\mathrm{CDCl}_{3}\right): \delta 7.58$ $7.55(\mathrm{~m}, 2 \mathrm{H}), 7.46-7.43(\mathrm{~m}, 2 \mathrm{H}), 3.73-3.69(\mathrm{~m}, 2 \mathrm{H}), 2.50-2.46(\mathrm{~m}, 2 \mathrm{H}), 1.79-1.65(\mathrm{~m}$, 4H), 1.40 (bs, $1 \mathrm{H}) .{ }^{13} \mathrm{C}$ NMR (100 MHz, $\left.\mathrm{CDCl}_{3}\right): \delta 132.3,132.1,129.2,118.8,110.8$, 95.5, 79.9, 62.2, 32.0, 25.0, 19.5. HRMS m/z Calcd for $\mathrm{C}_{13} \mathrm{H}_{13} \mathrm{NO}$ : 199.09971. Found: 199.10010. Anal. Calcd for $\mathrm{C}_{13} \mathrm{H}_{13} \mathrm{NO}$ : C, 78.39; H, 6.53. Found: C, 78.21; H, 6.59. 
2-(4-Cyanophenyl)-1-ethynyl(trimethyl)silane (Table 3, entry 6) ${ }^{1} \mathrm{H} \mathrm{NMR}\left(\mathrm{CDCl}_{3}\right)$ : $\delta$ 7.60-7.52 (m, 4H), $1.13(\mathrm{~s}, 21 \mathrm{H}) .{ }^{13} \mathrm{C} \mathrm{NMR}\left(\mathrm{CDCl}_{3}\right): \delta 132.7,132.1,128.5,118.7,105.3$, 96.5, 18.8, 11.4. HRMS m/z Calcd for $\mathrm{C}_{18} \mathrm{H}_{25} \mathrm{NSi}$ : 283.17563. Found: 283.17620. Anal. Calcd for $\mathrm{C}_{18} \mathrm{H}_{25} \mathrm{NSi}$ : C, 76.32; H, 4.59. Found: C, 76.08; H, 4.53.

4-Cyanodiphenylacetylene ${ }^{8}$ (Table 3, entry 7) ${ }^{1} \mathrm{H}$ NMR $\left(\mathrm{CDCl}_{3}\right): \delta 7.65-7.59(\mathrm{~m}, 4 \mathrm{H})$, 7.57-7.55 (m, 2H), 7.40-7.38 (m, 3H). ${ }^{13} \mathrm{C} \mathrm{NMR}\left(\mathrm{CDCl}_{3}\right): \delta 132.2,132.3,131.9,129.3$, $128.7,128.4,122.3,118.7,111.6,93.9,87.9$.

\section{References for known compounds:}

(1) Datta, A.; Plenio, H. Chem. Commun. 2003, 1504.

(2) Ma, Y.; Song, C.; Jiang, W.; Wu, Q.; Wang, Y.; Liu, X.; Andrus, M. B. Org. Lett. $\mathbf{2 0 0 3}, 5,3317$.

(3) Shen, W.; Wang. L. J. Org. Chem. 1999, 64, 8873.

(4) Nguefack, J.; Bolitt, V.; Sinou, D. Tetrahedron Lett. 1996, 37, 5527.

(5) Denmark, S. E.; Tymonko, S. A. J. Org. Chem. 2003, 68, 9151.

(6) Gapinski, M. D.; Mallett, B. E.; Froelich, L. L.; Jackson, W. T. J. Med. Chem. 1990, $33,2807$.

(7) Kollhofer, A.; Pullmann, T.; Plenio, H. Angew. Chem., Int. Ed. 2003, 42, 1056.

(8) Gelman, D.; Buchwald, S. L. Angew. Chem., Int. Ed. 2003, 42, 5993. 